\title{
An Assessment on the Hidden Ecological Factors of the Incidence of Malaria ${ }^{\dagger}$
}

\author{
Babagana Modu ${ }^{1, *}$, A. Taufiq Asyhari ${ }^{2}$, Savas Konur ${ }^{1}$ and Yonghong Peng ${ }^{3}$ \\ 1 School of Electrical Engineering and Computer Science, University of Bradford, Bradford BD7 1DP, UK; \\ s.konur@bradford.ac.uk \\ 2 Centre for Electronic Warfare, Information and Cyber, Cranfield University, Defense Academy, \\ Shrivenham SN6 8LA, UK; taufiq-a@ieee.org \\ 3 Faculty of Computer Sciences, University of Sunderland, St Peters Campus, Sunderland SR6 0DD, UK; \\ Yonghong.Peng@sunderland.ac.uk \\ * Correspondence: b.modu@bradford.ac.uk; Tel.: +44-7435-382-027 \\ + Presented at the IS4SI 2017 Summit DIGITALISATION FOR A SUSTAINABLE SOCIETY, Gothenburg, \\ Sweden, 12-16 June 2017.
}

Published: 9 June 2017

\begin{abstract}
Confounding effects of climatic factors temporally contribute to the prevalence of malaria. In this study, we explore a new framework for assessment and identification of hidden ecological factors to the incidence of malaria. A statistical technique, partial least squares path modeling and exploratory factor analysis, is employed to identify hidden ecological factors. Three hidden factors are identified: Factor I is related to minimum temperature and relative humidity, Factor II is related to maximum temperature and solar radiation and Factor III is related to precipitation and wind speed, respectively. Factor I is identified as the most influential hidden ecological factor of malaria incidence in the study area, as evaluated by communality and Dillon-Goldstein's indices.
\end{abstract}

Keywords: malaria incidence; climatic factors; structural equation modeling; partial least square model and hidden factors

\section{Introduction}

Malaria is a vector borne disease transmitted by female mosquito through sucking of human blood. According to the World Health Organization (WHO) statistics, about two-third of the world population are at risk of malaria, with resultant death close to a million annually [1]. The prevalence of malaria especially in tropical and sub-tropical regions is attributed to climatic factors and in some cases, human activities through poor sanitation, overwhelmed sewage and deforestation.

A recent study [2] showed that malaria incidence has significant correlation with relative humidity and whereas temperature and precipitation have negligible influence. The paper presented time-series modeling and lagged-regression of climate data together with reported malaria incidence cases. However, the methodology used fails to capture the existing dependency among the climate factors that normally exists.

In this study, we propose the use of partial least squares path modeling (PLS-PM) to analyze the causal relationship between meteorological variables such as: minimum average temperature, maximum average temperature, relative humidity, altitude, wind speed, precipitation and solar radiation, and explore their impacts on the occurrence of malaria incidence. By doing so, we aim to develop an integrated model that provides us with necessary information to identify the hidden ecological factors that lead to the high malaria incidence in the study area. 


\section{Results}

Three hidden factors were identified namely: Factor I (related to minimum temperature and relative humidity), Factor II (related to maximum temperature and solar radiation) and Factor III (related to precipitation and wind speed), respectively. The identified factors are accounted for $64 \%$ of the total variance, and at $\alpha=5 \%$ level of significance, $\chi^{2}=13.91, \mathrm{df}=8, p$-value $=0.0841$. This provides sufficient evidence to explain malaria incidence in the study area. Among the three factors, we find that Factor I, indicated by minimum temperature and relative humidity, influences malaria transmission as evaluated by communality index (0.94) and Dillon-Goldstein's @ (0.97). This result is also consistent with the findings in [3].

\section{Conclusions}

This study has provided an overview of ecosystem for malaria modeling and proposed a new framework for the study of malaria transmission ecosystem for prevention and control. Our data analysis results have suggested that the minimum temperature and relative humidity, which are related Factor I, have positive correlation with the incidence of malaria in the study area.

\section{References}

1. World Health Organization. Malaria Rapid Diagnostic Test Performance: Results of Who Product Testing of Malaria RDTs; WHO: Geneva, Switzerland, 2015.

2. Modu, B.; Asyhari, A.T.; Peng, Y.H. Data Analytics of Climatic Factor Influence on the Impact of the Malaria Incidence. In Proceedings of the IEEE Symposium Series on Computational Intelligence, Athens, Greece, 6-9 December 2016.

3. Xu, L.; Stige, L.C.; Chan, K.S.; Zhou, J.; Yang, J.; Sang, S.; Wang, M.; Yang, Z.; Yan, Z.; Jiang, T.; et al. Climate variation drives dengue dynamics. PANS 2016, 114, doi:10.1073/pnas.1618558114.

(C) 2017 by the authors. Licensee MDPI, Basel, Switzerland. This article is an open access article distributed under the terms and conditions of the Creative Commons Attribution (CC BY) license (http://creativecommons.org/licenses/by/4.0/). 